\title{
Ramanujan's theorem and highest abundant numbers
}

\author{
Oleg R. Musin
}

\begin{abstract}
In 1915, Ramanujan proved asymptotic inequalities for the sum of divisors function, assuming the Riemann hypothesis ( $\mathrm{RH})$. We consider a strong version of Ramanujan's theorem and define highest abundant numbers that are extreme with respect to the Ramanujan and Robin inequalities. Properties of these numbers are very different depending on whether the $\mathrm{RH}$ is true or false.
\end{abstract}

\section{Introduction}

The function $\sigma(n)=\sum_{d \mid n} d$ is the sum of divisors function. In 1913 Grönwall (see [5, Theorem 323]) proved that the asymptotic maximal size of $\sigma(n)$ satisfies

$$
\limsup _{n \rightarrow \infty} G(n)=e^{\gamma}, \quad G(n):=\frac{\sigma(n)}{n \log \log n}, n \geq 2,
$$

where $\gamma \approx 0.5772$ is the Euler-Mascheroni constant. Robin [11] showed that the Riemann hypothesis $(\mathrm{RH})$ is true if and only if

$$
\sigma(n)<e^{\gamma} n \log \log n \text { for all } n>5040 .
$$

Briggs' computation of the colossally abundant numbers implies $(\mathrm{R})$ for $n<10^{\left(10^{10}\right)}[2]$. According to Morrill and Platt [7], (R) holds for all integers $5040<n<10^{\left(10^{13}\right)}$.

A positive integer $n$ is called superabundant (SA) if

$$
\frac{\sigma(k)}{k}<\frac{\sigma(n)}{n} \text { for all integer } k \in[1, n-1] .
$$

Colossally abundant numbers (CA) are those numbers $n$ for which there is $\varepsilon>0$ such that

$$
\frac{\sigma(k)}{k^{1+\varepsilon}} \leq \frac{\sigma(n)}{n^{1+\varepsilon}} \text { for all } k \in \mathbb{N}
$$

Bachmann (see [5, Theorem 324]) showed that on average, $\sigma(n)$ is around $\pi^{2} n / 6$. Bachmann and Grönwall's results ensure that for every $\varepsilon>0$ the function $\sigma(n) / n^{1+\varepsilon}$ has a 
maximum and that as $\varepsilon$ tends to zero these maxima will increase. Thus there are infinitely many CA numbers.

SA and CA numbers were studied in detail by Alaoglu \& Erdős [1] and Erdős \& Nicolas [4]. The study of numbers with $\sigma(n)$ large was initiated by Ramanujan [9]. In fact, SA and CA numbers had been studied by Ramanujan in 1915. Unknown to Alaoglu and Erdös, about 30 pages of Ramanujan's paper "Highly Composite Numbers"' were suppressed. Those pages were finally published in 1997 [10].

Let

$$
\begin{gathered}
F(x, k):=\frac{\log \left(1+1 /\left(x+\ldots+x^{k}\right)\right)}{\log x}, \\
E_{p}:=\{F(p, k) \mid k \geq 1\}, \quad p \text { is prime, }
\end{gathered}
$$

and

$$
E:=\bigcup_{p} E_{p}=\left\{\varepsilon_{1}, \varepsilon_{2}, \ldots\right\}=\left\{\log _{2}\left(\frac{3}{2}\right), \log _{3}\left(\frac{4}{3}\right), \log _{2}\left(\frac{7}{6}\right), \ldots\right\} .
$$

Alaoglu and Erdös [1, Theorem 10] showed that if $\varepsilon$ is not critical, i.e. $\varepsilon \notin E$, then $\sigma(k) / k^{1+\varepsilon}$ has a unique maximum attained at the number $n_{\varepsilon}$. If $\varepsilon$ satisfies $\varepsilon_{i}>\varepsilon>\varepsilon_{i+1}, i \in \mathbb{N}$, then $n_{\varepsilon}$ is constant on the interval $\left(\varepsilon_{i+1}, \varepsilon_{i}\right)$ and we call it $n_{i}$. Moreover,

$$
n_{\varepsilon}=\prod_{p \in \mathbb{P}} p^{a_{\varepsilon}(p)}, \text { where } a_{\varepsilon}(p)=\left\lfloor\frac{\log \left(p^{1+\varepsilon}-1\right)-\log \left(p^{\varepsilon}-1\right)}{\log p}\right\rfloor-1 .
$$

In particular, Alaoglu and Erdös in their 1944 paper found all SA and CA numbers up to $10^{18}$. The first $14 \mathrm{CA}$ numbers $n_{1}, n_{2}, \ldots, n_{14}$ are

$$
2,6,12,60,120,360,2520,5040,55440,720720,1441440,4324320,21621600,367567200 .
$$

Robin [11, Sect. 3: Prop. 1] showed that if the Riemann hypothesis is false, then there exists a counterexample to the Robin criterion $(\mathrm{R})$ which is a colossally abundant number. Thus, it suffices to check (R) only for CA numbers.

Ramanujan, see [10, p. 143], proved that if $n$ is a CA number (he called CA numbers as generalized superior highly composite) then under the RH the following inequalities hold

$$
\begin{gathered}
\limsup _{n \rightarrow \infty}\left(\frac{\sigma(n)}{n}-e^{\gamma} \log \log n\right) \sqrt{\log n} \leq-c_{1}, c_{1}:=e^{\gamma}(2 \sqrt{2}-4-\gamma+\log 4 \pi) \approx 1.3932, \\
\liminf _{n \rightarrow \infty}\left(\frac{\sigma(n)}{n}-e^{\gamma} \log \log n\right) \sqrt{\log n} \geq-c_{2}, c_{2}:=e^{\gamma}(2 \sqrt{2}+\gamma-\log 4 \pi) \approx 1.5578
\end{gathered}
$$

Denote

$$
T(n):=\left(e^{\gamma} \log \log n-\frac{\sigma(n)}{n}\right) \sqrt{\log n}
$$

It is easy to see that Ramanujan's inequalities (1) and (2) yield the following fact: 
If the $R H$ is true, then there is $i_{0}$ such that for all CA numbers $n_{i}, i \geq i_{0}$, we have

$$
1.393<T\left(n_{i}\right)<1.558
$$

Note that (2) does not hold for all integers. Indeed, if $p_{i}$ is prime, then $\sigma\left(p_{i}\right)=p_{i}+1$. Therefore,

$$
\limsup _{i \rightarrow \infty} T\left(p_{i}\right)=\infty
$$

However, (1) holds for all numbers. In Section 2 we prove the following theorem.

Theorem 1 (The Strong Ramanujan Theorem). If the $R H$ is true, then

$$
\liminf _{n \rightarrow \infty} T(n) \geq c_{1}>1.393
$$

It is an interesting open problem: Can Ramanujan's constant $c_{1}$ be improved?

Theorem 1 implies the following inequality (see Corollary 1 in Section 2):

If the $R H$ is true, then there is $m_{0}$ such that for all $n>m_{0}$ we have

$$
\sigma(n)+\frac{1.393 n}{\sqrt{\log n}}<e^{\gamma} n \log \log n
$$

which is stronger than Ramanujan's theorem [3, Theorem 7.2]:

If the RH is true, then there is $m_{0}$ such that for all $n>m_{0}$ we have

$$
\sigma(n)<e^{\gamma} n \log \log n
$$

Note that, for fixed $\varepsilon>0$, CA numbers $n$ may be viewed as maximizers of

$$
Q(k)-\varepsilon \log k=\log \left(\sigma(k) / k^{1+\varepsilon}\right), \quad Q(k):=\log \sigma(k)-\log k .
$$

Equivalently, $n$ is CA if $\left(x_{n}, A\left(x_{n}\right)\right)$ is a vertex of the convex envelope of $A$ on $D$, where

$$
x_{k}:=\log k, \quad A\left(x_{k}\right):=x_{k}-\log \sigma(k)=-Q(k), \quad D:=\left\{x_{k}\right\}, \quad k \geq 2,
$$

see details in Section 3, Example 1.

Let $n \geq 2$ and $s$ be a real number. Denote

$$
R_{s}(n):=\left(e^{\gamma} n \log \log n-\sigma(n)\right)(\log n)^{s} .
$$

Now we define Highest Abundant (HA) numbers. We say that $n \in D \subset \mathbb{N}$ is $H A$ with respect to $R_{s}$ and write $n \in H A_{s}(D)$ if for some real a

$$
R_{s}(k)-a k
$$


attains its minimum on $D$ at $n$. For $D=\{n \in \mathbb{N} \mid n \geq 5040\}$ we denote $H A_{s}(D)$ by $H A_{s}$.

Actually, if $D$ is infinite, then $H A_{s}(D)$ can be empty or contain only one number $m_{0}$. It is clear that $m_{0}$ is the minimum number in $D=\left\{m_{0}=x_{0}, x_{1}, \ldots\right\}$. Then there is $a_{0}$ such that $m_{0}$ is defined by any $a \leq a_{0}$.

It can be shown, see Proposition 1 in Section 3, if $H A_{s}(D)=\left\{m_{0}, m_{1} \ldots\right\}$ contains at least two numbers, then there is a set of critical values $a, \mathcal{A}_{s}(D)=\left\{a_{1}, a_{2}, \ldots\right\}, a_{1}<a_{2}<\ldots$, such that if $a$ is not critical, then $R_{s}(n)-n a$ has a unique minimum on $D$ attained at the number $m_{a}$. If $a \in\left(a_{i}, a_{i+1}\right), i=1,2, \ldots$, then $m_{a}$ is constant on the interval $\left(a_{i}, a_{i+1}\right)$ and $m_{a}=m_{i}$. In fact, $a_{i}$ is the slope of $R_{s}$ on $\left[m_{i-1}, m_{i}\right]$, i.e.

$$
a_{i}=\frac{R_{s}\left(m_{i}\right)-R_{s}\left(m_{i-1}\right)}{m_{i}-m_{i-1}} .
$$

We see that definitions of CA and HA numbers are similar, in both cases numbers can be determined through the vertices of the convex envelopes of certain functions. In Example 3 (Section 3) is considered HA numbers with respect to $R_{s}, s=1$, on $D=\left[2, n_{13}=21621600\right]$. There are $13 \mathrm{HA}$ numbers in this interval, 12 of them are CA numbers (except $n_{6}=360$ ) and one more $m=2162160$ is SA but $m$ is not CA. However, properties of HA and CA numbers are different. The property that $H A_{s}$ is infinite depending on whether the $\mathrm{RH}$ is true or false.

Theorem 2. (i) Let $s>1 / 2$. If the $R H$ is true, then $H A_{s}$ is infinite and $\lim _{n \rightarrow \infty} a_{n}=\infty$. If the $R H$ is false, then $H A_{s}$ is empty.

(ii) Let $s \leq 0$. If the $R H$ is false, then $H A_{s}$ is infinite, all $a_{i}<0$ and $\lim _{n \rightarrow \infty} a_{n}=0$. If the $R H$ is true, then $H A_{s}=\{5040\}$ and $\mathcal{A}_{s}=\{0\}$.

In Section 4 (Theorems 3 and 4) we consider extensions of Theorem 2, Proofs of these theorems rely on Robin's inequalities (7) and (8) [Section 4], the strong Ramanujan theorem and his inequality (2), namely on Corollary 2 in Section 2.

Let $h_{n}:=\sum_{i=1}^{n} 1 / i$ denote the harmonic sum. Using (R) Lagarias [6] showed that the Riemann hypothesis is equivalent to the following inequality

$$
L_{0}(n):=h_{n}+\exp \left(h_{n}\right) \log \left(h_{n}\right)-\sigma(n)>0 \text { for all } n>1
$$

In Section 4 we consider an analog of Theorem 2 for $(\mathrm{L})$ on $D=\mathbb{N}$.

\section{The strong Ramanujan theorem}

Ramanujan's theorem in the form of (5) is present in [3, Theorem 7.2], [8], [10, p. 152] and other places. This theorem can be easily derived from (1) for the CA numbers. Theorem 1 extends (1) for all $n \in \mathbb{N}$ and is a strong version of Ramanujan's theorem, see (4). However, we could not find a proof of Theorem 1 for arbitrary integers. In this section we fill this gap. 
Proof of Theorem 1. Let

$$
f(n):=\sqrt{\log n} \log \log n, \quad g(n):=e^{\gamma}-G(n) .
$$

Then $T(n)=f(n) g(n)$.

Let $S$ be the set of all non-CA integers $n>2$. Then for every $n \in S$ there is $i=i(n)>1$ such that $n_{i-1}<n<n_{i}$, where $n_{i-1}$ and $n_{i}$ are two consecutive CA numbers. Robin [11, Proposition 1] showed that

$$
G(n) \leq \max \left(G\left(n_{i-1}\right), G\left(n_{i}\right)\right) .
$$

We divide $S$ into two disjoint subsets $S_{1}$ and $S_{2}$ :

$$
S_{1}:=\left\{n \in S \mid G(n) \leq G\left(n_{i-1}\right)\right\}, \quad S_{2}:=\left\{n \in S \mid G\left(n_{i-1}\right)<G(n) \leq G\left(n_{i}\right)\right\} .
$$

(1) Suppose $n \in S_{1}$. Then $g(n) \geq g\left(n_{i-1}\right)$, where $i=i(n)$. Since $f$ is a monotonically increasing function, we have $f(n)>f\left(n_{i-1}\right)$ and $T(n)>T\left(n_{i-1}\right)$. Thus,

$$
\liminf _{n \in S_{1}, n \rightarrow \infty} T(n) \geq \liminf _{i \rightarrow \infty} T\left(n_{i-1}\right)=\liminf _{i \rightarrow \infty} T\left(n_{i}\right) \geq c_{1} .
$$

(2) Suppose $n \in S_{2}$. Then $g(n) \geq g\left(n_{i}\right)$ and $f(n)>f\left(n_{i-1}\right)$. That yields

$$
T(n)>f\left(n_{i-1}\right) g\left(n_{i}\right)=T\left(n_{i}\right) F(i), \quad F(i):=\frac{f\left(n_{i-1}\right)}{f\left(n_{i}\right)} .
$$

We have

$$
\lim _{i \rightarrow \infty} \frac{\log \left(n_{i-1}\right)}{\log \left(n_{i}\right)}=1
$$

Indeed, let $P(n)$ denote the largest prime factor of $n$. Alaoglu \& Erdös [1, Theorem 7] proved that $P(n) \sim \log n$ for all SA numbers. Then, in particular, it holds for CA numbers. The quotient of two consecutive CA numbers is either a prime or the product of two distinct primes [1, page 455], [3, Lemma 6.15], i.e. $n_{i} \leq n_{i-1} P^{2}\left(n_{i}\right) \sim n_{i-1} \log ^{2}\left(n_{i}\right)$. Then we have

$$
1>\frac{\log \left(n_{i-1}\right)}{\log \left(n_{i}\right)}>\frac{\log \left(n_{i}\right)-2 \log \left(P\left(n_{i}\right)\right)}{\log \left(n_{i}\right)} \sim 1-\frac{2 \log \log n_{i}}{\log n_{i}} \sim 1 .
$$

It is not hard to see that (6) implies $\lim _{i \rightarrow \infty} F(i)=1$. That yields

$$
\liminf _{n \in S_{2}, n \rightarrow \infty} T(n) \geq \liminf _{i \rightarrow \infty} T\left(n_{i}\right) F(i)=\liminf _{i \rightarrow \infty} T\left(n_{i}\right) \geq c_{1}
$$

Thus, we have (1) for CA, $S_{1}$ and $S_{2}$, i.e. for all numbers.

Remark. In the first version of this paper our proof of Case (2) relies on [12, Theorem 1]. I am very grateful to Xiaolong $\mathrm{Wu}$ for the idea of proving this case using (6). Note that (6) is easily derived from the results of the classical paper of Alaoglu and Erdős [1]. 
Corollary 1. If the $R H$ is true, then for every $\varepsilon>0$ there is $m_{0}$ such that for all $n>m_{0}$ we have

$$
\sigma(n)+\left(c_{1}-\varepsilon\right) \frac{n}{\sqrt{\log n}}<e^{\gamma} n \log \log n .
$$

In particular, if $\varepsilon \leq 1.393$, then $\sigma(n)<e^{\gamma} n \log \log n$ for all $n>m_{0}$.

From (2) for CA numbers $n_{i}$ we have

$$
\limsup _{i \rightarrow \infty} T\left(n_{i}\right) \leq c_{2}<1.558 .
$$

This fact and Corollary 1 yield the following corollary:

Corollary 2. If the $R H$ is true, then for every $\varepsilon>0$ there is $m_{0}$ such that a set

$$
M(\varepsilon):=\left\{n>m_{0} \mid T(n)<c_{2}+\varepsilon\right\}
$$

is infinite and for all $n \in M(\varepsilon)$ we have $T(n)>c_{1}-\varepsilon$.

\section{Convex envelope of functions}

Let $D=\left\{x_{n}\right\}$ be an increasing sequence. Let $h: D \rightarrow \mathbb{R}$ be a function on $D$. We say that $h$ is convex (or concave upward) on $D$ if for all $a, x, b \in D$ such that $a<x<b$ we have

$$
h(x) \leq \frac{(b-x) h(a)+(x-a) h(b)}{b-a} .
$$

Denote by $\Omega(f)$ the set of all convex functions $h: D \rightarrow \mathbb{R}$ such that $h(x) \leq f(x)$ for all $x \in D$. Suppose $\Omega(f) \neq \emptyset$. The lower convex envelope $\breve{f}$ of a function $f$ on $D$ is defined at each point of $D$ as the supremum of all convex functions that lie under that function, i.e.

$$
\breve{f}(x):=\sup \{h(x) \mid h \in \Omega(f)\} .
$$

Alternatively, $\breve{f}$ can be defined as follows. Let

$$
\Gamma_{f}:=\left\{(x, f(x)) \in D \times \mathbb{R} \subset \mathbb{R}^{2}\right\}
$$

be the graph of $f$. The convex hull of $\Gamma_{f}$ in $\mathbb{R}^{2}$ is the set of all convex combinations of points in $\Gamma_{f}$ :

$$
\operatorname{conv}\left(\Gamma_{\mathrm{f}}\right):=\left\{\mathrm{c}_{1} \mathrm{p}_{1}+\ldots+\mathrm{c}_{\mathrm{k}} \mathrm{p}_{\mathrm{k}} \mid \mathrm{p}_{\mathrm{i}} \in \mathrm{G}_{\mathrm{f}}, \mathrm{c}_{\mathrm{i}} \geq 0, \mathrm{i}=1, \ldots, \mathrm{k}, \mathrm{c}_{1}+\ldots+\mathrm{c}_{\mathrm{k}}=1\right\} .
$$

Then the graph $\{(x, \breve{f}(x)) \in D \times \mathbb{R}\}$ is the lower convex hull of $\operatorname{conv}\left(\Gamma_{\mathrm{f}}\right)$.

It is clear, if $D$ is finite, then $\Omega(f)$ is not empty. However, if $D$ is infinite, then $\Omega(f)$ can be empty, for instance if $f(n)=-n^{2}$ and $D=\mathbb{N}$. 


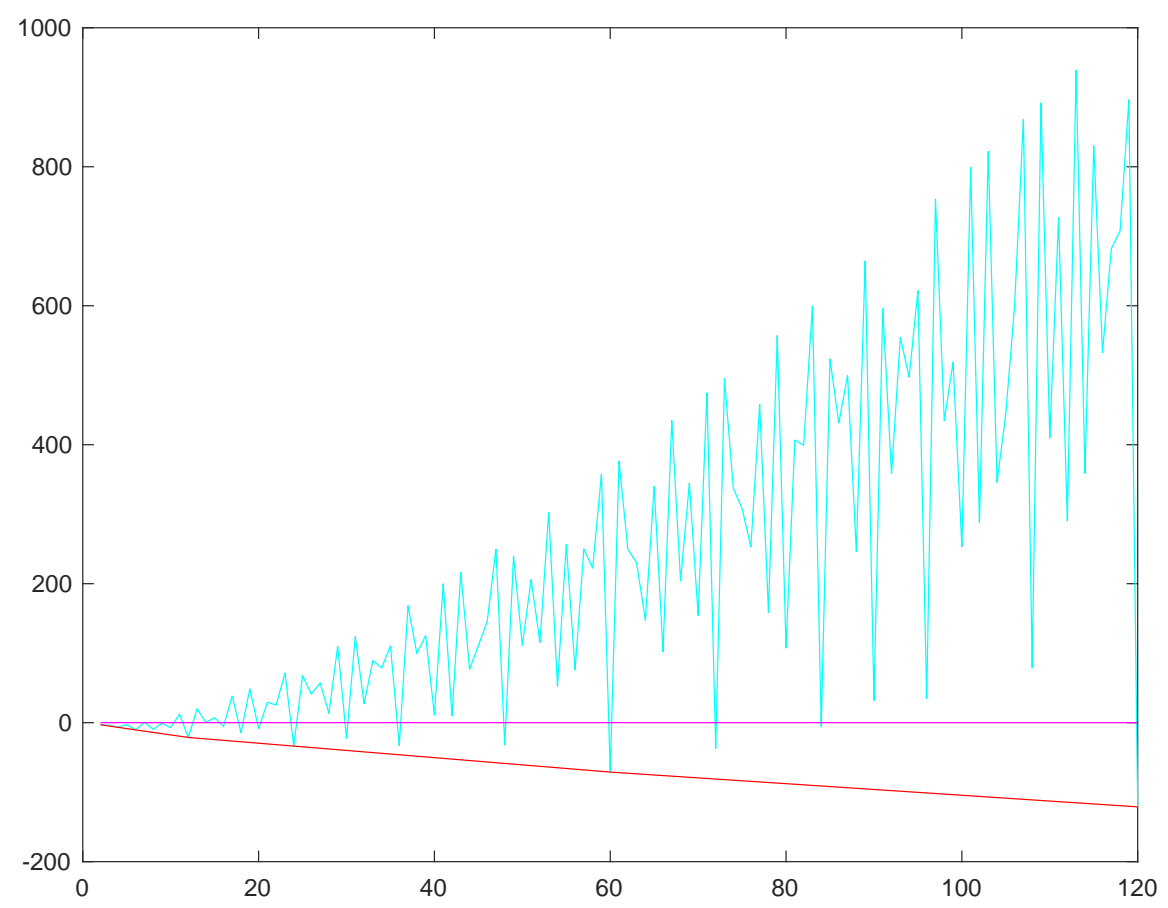

Figure 1: Graphs of $R_{1}$ and $\breve{R}_{1}$ on $D=\{2, \ldots, 120\}$.

Let $f$ be a function on $D=\left\{x_{0}, x_{1}, \ldots\right\}$ with $\Omega(f) \neq \emptyset$. Then $\breve{f}$ is a piecewise linear convex function on $D$. Hence, there is a subset

$$
H_{f}:=\left\{m_{0}=x_{0}, m_{1}, \ldots\right\} \subset D
$$

such that $\breve{f}$ is a linear function on $\left[m_{i-1}, m_{i}\right], \breve{f}\left(m_{i}\right)=f\left(m_{i}\right)$ for all $i$, and the sequence of slopes $A_{f}:=\left\{a_{1}, a_{2}, \ldots\right\}$ is strictly monotonic increasing, i.e. $a_{1}<a_{2}<\ldots$, where

$$
a_{i}:=\frac{f\left(m_{i}\right)-f\left(m_{i-1}\right)}{m_{i}-m_{i-1}} .
$$

Let $\tilde{H}_{f}$ be a subset in $D$ such that $m \in \tilde{H}_{f}$ if for some $a \in \mathbb{R}$ the function $f(x)-a x$ attains its minimum on $D$ at $m$, i.e.

$$
\tilde{H}_{f}:=\{m \in D \mid \exists a \in \mathbb{R}, \forall x \in D, f(m)-m a \leq f(x)-a x\} .
$$

The next proposition can be easily derived from the above definitions.

Proposition 1. Let $f$ be a function on $D=\left\{x_{n}\right\}$ with $\Omega(f) \neq \emptyset$. Then $\tilde{H}_{f}$ coincides with $H_{f}$ and every $m_{i} \in H_{f}, i \geq 1$, is uniquely determined by any $a \in\left(a_{i-1}, a_{i}\right)$. 
Example 1. Let $D:=\left\{x_{n}\right\}$, where $x_{n}:=\log n, n \in \mathbb{N}$. Let $f\left(x_{n}\right):=x_{n}-\log \sigma(n)$. Then $f\left(x_{n}\right)=-Q(n)$, where $Q$ is defined in Section 1. It is easy to see that in this case Proposition 1 yields that $H_{f}$ is the set of CA numbers and $A_{f}=\left\{-\varepsilon_{i}\right\}$.

If $D=\left\{x_{0}, x_{1}, \ldots, x_{l}\right\}$ is finite, then $H_{f}:=\left\{m_{0}=x_{0}, m_{1}, \ldots, m_{k} \leq x_{l}\right\}$ and the cardinality $\left|A_{f}\right|=k$. If $D$ is infinite, then $A_{f}$ can be (i) infinite or (ii) finite. It is not hard to see that in case (ii) $H_{f}:=\left\{m_{0}, m_{1}, \ldots, m_{k}\right\}$ and $A_{f}=\left\{a_{1}, \ldots, a_{k}, a_{k+1}\right\}$, where

$$
\breve{f}(n)=\breve{f}\left(m_{k}\right)+\left(n-m_{k}\right) a_{k+1} \text { for all } n \in D, n \geq m_{k} \text {. }
$$

Let $m_{k+1}:=\infty$. Then for both cases we have that $a_{i}$ is the slope of $\breve{f}$ on $\left[m_{i-1}, m_{i}\right]$.

Example 2. Let $f(n)=R_{1}(n)=\left(e^{\gamma} n \log \log n-\sigma(n)\right) \log n$ on $D=\{2, \ldots, 120\}$. Then $H_{f}=\{2,6,12,60,120\}$. (Note that $H_{f}$ consists of the first five CA numbers.) In this case $\breve{R}_{1}$ is a convex monotonically decreasing function on $D$, see Figure 1 .

Example 3. Let $f=R_{1}$ on $D=\left\{2,3, \ldots, n_{13}=21621600\right\}$. Then

$$
H_{f}=\{2,6,12,60,120,2520,5040,55440,720720,1441440,2162160,4324320,21621600\} .
$$

In this list of 13 numbers $m_{0}, \ldots, m_{12}$ there are 12 out of the first $13 \mathrm{CA}$ numbers except $n_{6}=360$. However, $m_{10}$ is an SA number $2162160=2^{4} \cdot 3^{3} \cdot 5 \cdot 7 \cdot 11 \cdot 13$ but is not CA. $\breve{R}_{1}$ on $H_{f}$ has a minimum at $m_{5}=2520$ and is positive for $m_{i}>m_{6}=5040$. We have

$$
a_{1}<\ldots<a_{5}<0<a_{6}<\ldots<a_{12} .
$$

Now we prove the main results of this section.

Lemma 1. Let $D \subset \mathbb{N}$ be infinite and $n_{0} \in D$. Let $f$ and $g$ be functions on $D$ such that

$$
f(n) \geq g(n) \text { for all } n \geq n_{0} \text { and } \lim _{n \rightarrow \infty} \frac{g(n)}{n}=\infty .
$$

Then $A_{f}$ is infinite and $\lim _{n \rightarrow \infty} a_{n}=\infty$.

Proof. By assumption, for any real $a$ there is $n_{a}$ such that $g(n)>$ an for all $n \geq n_{a}$. This fact yields that for any linear function $l(x)=a x+b$ there is no or there are finitely many $n \in D$ such that $f(n) \leq l(n)$.

Let $D=\left\{x_{0}, x_{1}, \ldots\right\}, m_{0}:=x_{0}$ and $H_{f}^{(0)}:=\left\{m_{0}\right\}$. Suppose $H_{f}^{(i)}=\left\{m_{0}, \ldots, m_{i}\right\}$ and $\mathcal{A}_{f}^{(i)}=\left\{a_{1}, \ldots, a_{i}\right\}$. Let $l(x)$ be a linear function given by two points $\left(m_{i}, f\left(m_{i}\right)\right)$ and $\left(x_{k+1}, f\left(x_{k+1}\right)\right)$, where $m_{i}=x_{k}$. Denote

$$
D_{l}=\left\{n \in D \mid n>m_{i}, f(n) \leq l(n)\right\} .
$$

We have $1 \leq\left|D_{l}\right|<\infty$. Let $x_{j}$ be a number in $D_{l}$ such that the slope of a linear function given by two points $\left(m_{i}, f\left(m_{i}\right)\right)$ and $(n, f(n)), n \in D_{l}$, attains its minimum at $x_{j}$. We denote the correspondent linear function by $l_{i+1}$. It is clear that $f(n) \geq l_{i+1}(n)$ for all $n \in D_{l}$. Hence, $m_{i+1}=x_{j}$ and $a_{i+1}$ is the slope of $l_{i+1}$. We can continue this process. Since $f(n) / n \rightarrow \infty$ as $n \rightarrow \infty$, we have $a_{i} \rightarrow \infty$ as $i \rightarrow \infty$. 
Lemma 2. Let $g_{1}$ and $g_{2}$ be functions on $D \subset \mathbb{N}$ such that for all $n \in D$ we have

$$
g_{2}(n) \geq g_{1}(n) \text { and } \lim _{n \rightarrow \infty} g_{1}(n)=\infty, \quad \lim _{n \rightarrow \infty} \frac{g_{2}(n)}{n}=0 .
$$

Suppose for a function $f$ on $D$ there is $n_{0} \in D$ such that $f(n) \geq g_{1}(n)$ for all $n \geq n_{0}$. If there are infinitely many $n \in D$ such that $f(n) \leq g_{2}(n)$, then $A_{f}=\left\{a_{1}, \ldots, a_{k}\right\}$ is finite and

$$
a_{1}<\ldots<a_{k}=0 .
$$

Proof. Denote $D_{0}:=\left\{n \in D \mid n<n_{0}\right\}$ and $D_{1}:=\left\{n \in D \mid n \geq n_{0}, f(n) \leq g_{2}(n)\right\}$ By assumption, $D_{1}$ is infinite and for any linear function $l(x)=a x+b$ with $a>0$ there is no or there are finitely many $n \in D_{1}$ such that $f(n) \geq l(n)$. Hence, all $a_{i} \leq 0$. Since $f(n) \rightarrow \infty$ as $n \rightarrow \infty$, we have that $A_{f}$ is finite and the largest $a_{k}=0$.

Lemma 3. Let $g_{1}$ and $g_{2}$ be functions on $D \subset \mathbb{N}$ such that for all $n \in D$ we have

$$
g_{2}(n) \geq g_{1}(n) \text { and } \lim _{n \rightarrow \infty} g_{2}(n)=-\infty, \quad \lim _{n \rightarrow \infty} \frac{g_{1}(n)}{n}=0 .
$$

Suppose for a function $f$ on $D$ there is $n_{0} \in D$ such that $f(n) \geq g_{1}(n)$ for all $n \geq n_{0}$. If there are infinitely many $n \in D$ such that $f(n) \leq g_{2}(n)$, then $A_{f}$ is infinite and $\lim _{n \rightarrow \infty} a_{n}=0$.

Proof. It is not hard to see that the assumptions yield that for any $l(x)=a x+b$ with $a<0$ there is no or there are finitely many $n \in D$ such that $f(n) \leq l(n)$. Let $l_{i}$ be the same as in Lemma 1. In this case for $n \in D_{1}$, that defined in Lemma 2, we have $f(n) \rightarrow-\infty$ and $f(n) / n \rightarrow 0$ as $n \rightarrow \infty$. Thus, $a_{i} \rightarrow 0$ as $i \rightarrow \infty$.

Lemma 4. Let $D \subset \mathbb{N}$ be infinite. Let $g$ be a function on $D$ such that

$$
\lim _{n \rightarrow \infty} \frac{g(n)}{n}=-\infty
$$

Suppose for a function $f$ on $D$ there are infinitely many $n \in D$ such that $f(n) \leq g(n)$. Then $\Omega(f)$ is empty.

Proof. Let $D_{g}:=\{n \in D \mid f(n) \leq g(n)\}$. Let $l_{n}$ be a linear function given by two points $\left(x_{0}, f\left(x_{0}\right)\right)$ and $(n, f(n))$. By assumption for any $a$ there is $n \in D_{g}$ such that the slope of $l_{n}$ is less than $a$. Moreover, there are infinitely many $m$ in $D_{g}$ with $f(m)<l_{n}(m)$. This completes the proof.

\section{Proof of Theorem 2 and its extensions}

Robin [11, Theorem 2] showed that for all $n \geq 3$

$$
R_{0}(n)=e^{\gamma} n \log \log n-\sigma(n)>-0.6482 \frac{n}{\log \log n} .
$$


If the RH is false Robin [11, Theorem 1] proved that there exist constants $b \in(0,1 / 2)$ and $c>0$ such that

$$
R_{0}(n)<-\frac{c n \log \log n}{(\log n)^{b}}
$$

holds for infinitely many $n$. Thus, if the $\mathrm{RH}$ is false there are infinitely many $n \in \mathbb{N}$ such that

$$
C_{1}(n):=-\frac{0.6482 n}{\log \log n}<R_{0}(n)<C_{2}(n):=-\frac{c n \log \log n}{(\log n)^{b}} .
$$

Let $\tau(n)$ be any positive function on $D \subset \mathbb{N}$. Denote

$$
R_{\tau}(n):=\left(e^{\gamma} n \log \log n-\sigma(n)\right) \tau(n), n \in D .
$$

We defined HA numbers with respect to $R_{\tau}$ as follows:

$$
H A_{\tau}(D):=H_{R_{\tau}}(D)=\left\{m \in D \mid \exists a \in \mathbb{R}, \forall x \in D, R_{\tau}(m)-m a \leq R_{\tau}(x)-a x\right\} .
$$

As above, $\mathcal{A}_{\tau}(D)=\left\{a_{1}, a_{2}, \ldots\right\}$ are slopes of $R_{\tau}$ on $H A_{\tau}(D)$ and we denote $H A_{\tau}(D)$ by $H A_{\tau}$ for $D=\{n \in \mathbb{N} \mid n \geq 5040\}$.

The following theorem extends Theorem 2 (i).

Theorem 3. Let $\tau(n)>0$ for all $n \geq 5040$. Denote

$$
\Phi_{\tau}:=\lim _{n \rightarrow \infty} \frac{\tau(n)}{\sqrt{\log n}} .
$$

(a) Assume the RH is true. If $\Phi_{\tau}=\infty$, then $H A_{\tau}$ is infinite and $\lim _{n \rightarrow \infty} a_{n}=\infty$.

(b) If the $R H$ is false and $\Phi_{\tau}>0$, then $H A_{\tau}$ is empty.

Proof. (a) Suppose the RH is true. Let

$$
g(n):=\frac{1.393 n \tau(n)}{\sqrt{\log n}}
$$

By Corollary 1 there is $n_{0}$ such that for all $n \geq n_{0}$ we have

$$
R_{\tau}(n)=\frac{n T(n) \tau(n)}{\sqrt{\log n}} \geq g(n) \text { and by assumption } \lim _{n \rightarrow \infty} \frac{g(n)}{n}=1.393 \Phi_{\tau}=\infty .
$$

Then Lemma 1 with $f=R_{\tau}$ yields that $\lim _{n \rightarrow \infty} a_{n}=\infty$.

(b) Suppose the RH is false. Since $b<1 / 2$ by (8) there are infinitely many $n \in \mathbb{N}$ such that

$$
R_{\tau}(n)=R_{0}(n) \tau(n) \leq C_{2}(n) \tau(n)<g(n):=-\frac{c n \tau(n) \log \log n}{\sqrt{\log n}} .
$$

Then Lemma 4 with $f=R_{\tau}$ completes the proof. 
Now we consider a generalization of Theorem 2(ii).

Theorem 4. Let

$$
\tau(n)>0, n \geq 5040, \quad \lim _{n \rightarrow \infty} \frac{\tau(n)}{\log \log n}=0 \quad \text { and } \quad \lim _{n \rightarrow \infty} \frac{\tau(n) n \log \log n}{\sqrt{\log n}}=\infty .
$$

(a) If the $R H$ is false, then $H A_{\tau}$ is infinite, all $a_{i}<0$ and $\lim _{n \rightarrow \infty} a_{n}=0$.

(b) If the RH is true, then $H A_{\tau}=\{5040\}$ and $\mathcal{A}_{\tau}=\{0\}$.

Proof. (a) Suppose the RH is false. Let

$$
g_{1}(n):=C_{1}(n) \tau(n), \quad g_{2}(n):=C_{2}(n) \tau(n) .
$$

Then by (7) we have that $g_{1}(n)<R_{\tau}(n)$ for all $n \in D$ and by (8) the inequality $R_{\tau}(n)<g_{2}(n)$ holds for infinitely many $n$. Since $f=R_{\tau}, g_{1}$ and $g_{2}$ satisfy the assumption of Lemma 3 we have (a).

(b) Suppose the RH is true. Let

$$
g_{1}(n):=\frac{1.393 n \tau(n)}{\sqrt{\log n}}, \quad g_{2}(n):=\frac{1.558 n \tau(n)}{\sqrt{\log n}} .
$$

Then Corollary 2 yields that $f=R_{\tau}, g_{1}$ and $g_{2}$ satisfy the assumption of Lemma 2. Since for all $n>5040$ we have $R_{\tau}(n)>0>R_{\tau}(5040)$, there are not $a_{i} \leq 0$. Thus, $H A_{\tau}=\{5040\}$.

Proof of Theorem 2. This theorem immediately follows from Theorems 3 and 4 . Indeed, if $\tau(n)=(\log n)^{s}$, then $R_{\tau}(n)=R_{s}(n)$. It clear that $\Phi_{\tau}=\infty$ in Theorem 3 only if $s>1 / 2$ and the assumptions in Theorem 4 hold if $s \leq 0$.

From the Lagarias inequalities [6. Lemmas 3.1, 3.2] for $n>20$ we have

$$
R_{0}(n)+h_{n} \leq L_{0}(n) \leq R_{0}(n)+\frac{7 n}{\log n} .
$$

Let $L_{\tau}(n):=L_{0}(n) \tau(n)$. Then (9) yields analogs of Theorems 3 and 4 for $L_{\tau}$. We can just substitute $R_{\tau}$ by $L_{\tau}$.

Theorem 5. (i) If the RH is true, $\tau(n)>0$ and $\Phi_{\tau}=\infty$, then there are infinitely many $H A$ numbers with respect to $L_{\tau}$ and $\lim _{n \rightarrow \infty} a_{n}=\infty$. .

(ii) Let $\tau(n)$ satisfy the assumptions of Theorem 4. If the RH is false, then there are infinitely many $H A$ numbers with respect to $L_{\tau}$, all $a_{i}<0$ and $\lim _{n \rightarrow \infty} a_{n}=0$. 


\section{References}

[1] L. Alaoglu and P. Erdős, On Highly Composite and Similar Numbers, Trans. Amer. Math. Soc., 56 (1944), 448-469.

[2] K. Briggs, Abundant numbers and the Riemann hypothesis. Experiment. Math., 15 (2006), 251-256.

[3] K. Broughan, Equivalents of the Riemann Hypothesis. Cambridge Univ. Press, 2017.

[4] P. Erdős and J.-L. Nicolas, Répartition des nombres superabondants, Bull. Math. Soc. France, 103 (1975), 65-90.

[5] G. H. Hardy and E. M. Wright, An Introduction to the Theory of Numbers. Fifth Edition. Oxford Univ. Press: Oxford 1979.

[6] J. C. Lagarias. An Elementary Problem Equivalent to the Riemann Hypothesis. Am. Math. Monthly, 109 (2002), 534-543.

[7] T. Morrill, D. Platt. Robin's inequality for 25-free integers and obstacles to analytic improvement, preprint, arXiv:1809.10813

[8] J.-L. Nicolas and J. Sondow. Ramanujan, Robin, Highly Composite Numbers, and the Riemann Hypothesis, Contemp. Math., 627 (2014), 145-156.

[9] S. Ramanujan, Highly Composite Numbers, Proc. London Math. Soc., 14 (1915), 347407.

[10] S. Ramanujan, Highly Composite Numbers (annotated and with a foreword by J.-L. Nicolas and G. Robin), Ramanujan J., 1 (1997), 119-153.

[11] G. Robin. Grandes valeurs de la fonction somme des diviseurs et hypothése de Riemann. J. Math. Pures Appl., 63 (1984), 187-213.

[12] X. Wu, Subsets of colossally abundant numbers, preprint, arXiv:1903.03490

O. R. Musin, School of Mathematical and Statistical Sciences, University of Texas Rio Grande Valley, One West University Boulevard, Brownsville, TX, 78520.

E-mail address: omusin@gmail.com 